Психология. Журнал Высшей школы экономики.

2021. T. 18. № 1. C. 92-108. DOI: 10.17323/1813-8918-2021-1-92-108

\title{
МОТИВЫ ИННОВАТОРОВ В ОБРАЗОВАНИИ: ПОДХОДЫ К ОПРЕДЕЛЕНИЮ И РАЗРАБОТКА ШКАЛЫ ДЛЯ ИЗМЕРЕНИЯ
}

\author{
Т.Е. ХАВЕНСОН ${ }^{\mathrm{a}}$, А.А. ЛУКИНА \\ ${ }^{a}$ Национальный исследовательский университет «Высшая школа экономики», 101000, Россия, \\ Москва, ул. Мясниикая, д. 20
}

\section{Резюме}

Причины, по которым внутри и вне системы образования появляются инновации, способные существенно изменить или улучшить традиционно закрытую сферу, активно изучаются в последние десятилетия. Однако ощущается дефицит разработанных методик для измерения мотивации внешних и внутренних акторов создавать инновационные проекты. В статье описываются подходы к измерению самого конструкта «мотивация инновационной деятельности» и результаты адаптации инструмента для измерения мотивов создания инновационных проектов в сфере образования. За основу была взята англоязычная шкала «Причины основания бизнеса» (PSED). В рамках исследования шкала была переведена на русский язык, адаптирована для группы инноваторов в образовании и валидизирована с использованием классического подхода, сочетающего методологию разведывательного и конфирматорного факторного анализа (РФА и КФА), а также дополнительную оценку внутренней согласованности выявленных подшкал, сравнение оригинальной англоязычной и русскоязычной версий, содержательную интерпретацию каждой подшкалы. Адаптация шкалы проводилась на выборке инноваторов в сфере образования - участников Конкурса инноваций в образовании $(\mathrm{N}=286)$. Итоговая шкала включает 16 суждений и позволяет оценить выраженность четырех мотивационных установок начала инновационной деятельности: «Значимость для общества», «Инновации и креативность», «Самореализация и достижение» и «Финансы и независимость». Выявленные мотивы отражают определенные стремления и цели инноваторов, задавая содержание и направленность их образовательных проектов. Предлагаемый инструмент может быть использован в исследовательских и практических целях для изучения мотивации проактивных акторов в образовании, а также близких групп, вовлеченных в развитие социально-значимых сфер. Полная версия итоговой методики приведена в Приложении к статье, содержит инструкции для респондентов и правила подсчета баллов по 4 подшкалам.

Ключевые слова: мотивация к инновациям, PSED, адаптация методики, измерение мотивов инновационной деятельности.

\section{Введение}

Современные тренды развития общества, экономики и, как следствие, системы образования вызывают необходимость существенных изменений образовательных институтов. Важную роль в этом процессе приобретают

Публикация подготовлена при поддержке РФФИ, проект № 17-03-00837. 
индивидуальные игроки образовательного поля, именуемые также «низовыми инноваторами» (от англ. grassroots - низовой, массовый),- это люди, которые создают собственные независимые проекты, отталкиваясь от проблем и вопросов, возникающих в локальном контексте (OECD, 2014; Seyfang, Smith, 2007; Shirley, 2017; Кузьминов, Фрумин, 2018). Низовые инновации (или инновации снизу) обладают большим потенциалом для трансформации социальных институтов, поскольку способны оперативно реагировать на актуальные вызовы, заполнять существующие дефициты (Phelps, 2018) и изменять общепринятые нормы и практики (Battilana et al., 2009).

В России, несмотря на закрытость системы образования, низовые инновации пронизывают разные сферы образования, включая все ступени формального и сегменты дополнительного образования (Королева и др., 2017). Проекты возникают как внутри системы - их создают учителя, директора школ, преподаватели вузов и др., - так и за ее пределами (через деятельность родителей, студентов, предпринимателей). Их появление - это индикатор активности на местах и формирования поля новых перспективных разработок в сфере образования, направленных на обновление системы.

Важной характеристикой низовых инноваций является личная мотивация инноваторов: в отличие от формальной рабочей или учебной ситуации, которая предполагает превалирование интересов организации над индивидуальными целями, низовые инновации создаются добровольно и целиком зависят от их создателя, что открывает возможности для реализации многообразия мотивационных установок (Хавенсон и др., 2018). От мотивации инноваторов во многом зависят направленность и содержание низовых инноваций (Elfving, 2008; Jones et al., 2021), которые, в свою очередь, формируют ландшафт современного образования и задают направление его дальнейшего развития. В целом социально-психологический портрет инноватора, важным компонентом которого является мотивация, играет ключевую роль для проектов на ранней стадии развития (Frese, Gielnik, 2014). В этой связи мотивационные установки низовых инноваторов становятся важным объектом для изучения. Понимание этих установок позволит учитывать их в современной образовательной политике, разрабатывать релевантные механизмы их поддержки и в целом формировать среду, необходимую для развития инноваций. Необходимость изучения мотивации низовых инноваторов предполагает разработку соответствующих методик, учитывающих особенности этой группы.

Целью настоящего исследования является разработка методики для изучения мотивации инноваторов в сфере образования. Для этого была осуществлена адаптация шкалы «Причины начала бизнес-проектов», созданной в рамках американского исследования динамики предпринимательства (Panel Study of Entrepreneurial Dynamics, PSED; Carter et al., 2004). В отличие от других методик, направленных на измерение предпринимательских намерений (Ajzen, 1991; Shapero, Sokol, 1982), степени детерминированности деятельности и соотношения внутренней и внешней мотивации (Deci, Ryan, 1985), данный инструмент позволяет изучить содержательные аспекты мотивации инноваторов через измерение конкретной направленности их деятельности. 
Отдельные элементы опросника PSED часто применяются в современных исследованиях, апробированы и валидизированы для разных стран. В частности, они использовались для идентификации социальных предпринимателей (Quigley et al., 2015), изучения предпринимательских намерений студентов (Богатырева, Широкова, 2017) и мотивации социальных предпринимателей (Xu et al., 2014). Однако именно мотивационная компонента опросника PSED не валидизирована и не переведена на русский язык.

\section{Обзор подходов к измерению мотивов начала новых проектов}

В отсутствие необходимых измерительных инструментов, адаптированных для инноваторов в сфере образования, представляется релевантным рассмотреть ключевые подходы к измерению мотивов в психологии и предпринимательских исследованиях, поскольку инновационная деятельность в образовании может считаться частным случаем предпринимательской активности (Attali, Yemini, 2017).

В работах по изучению мотивации превалирует теория самодетерминации (self-determination theory) (Deci, Ryan, 1985), согласно которой мотивация варьируется по степени самостоятельности и независимости поведения. Опорными точками на этом континууме выступают три типа мотивации: внутренняя, внешняя мотивация и амотивация. Эта теория позволяет определить степень интернализации мотивации и режим регуляции действий, однако не учитывает конкретной направленности поведения, его содержательных причин.

В литературе по проблемам предпринимательства начало деятельности также изучается с точки зрения внешней и внутренней мотивации. В этой связи можно выделить две традиции: теории побуждения (drive theories) и теории поощрения (incentive theories) (Carsrud, Brännback, 2011). Теории побуждения исходят из того, что ключевым драйвером начала собственного бизнес-проекта является внутренняя потребность (например, достижения или независимости). Теории поощрения, напротив, рассматривают предпринимательскую активность как мотивированную возможным вознаграждением (доход, мобильность, престиж).

Однако последние исследования указывают на необходимость сочетать эти подходы, оставляя позади традицию одностороннего рассмотрения мотивации, поскольку бизнес-активность часто мотивирована сочетанием целей и имеет сразу несколько направлений (Caringal-Go, Hechanova, 2018; Ganguli et al., 2018; Перикова и др., 2020). Как показывают исследования, деятельность социальных предпринимателей мотивирована комбинацией экономических и неэкономических причин, причем ключевая роль отводится последним (Douglas, Prentice, 2019; Seelos, Mair, 2005; Zahra et al., 2009).

Близким направлением исследований бизнес-мотивации является изучение предпринимательских намерений: индивидуальных факторов, предшествующих решению открыть свое дело. Традиционными считаются две объяснительные модели: теория намеренного поведения (Theory of Planned 
Behavior; Ajzen, 1991, 2002) и модель предпринимательских намерений (Entrepreneurial Intentions Model; Shapero, Sokol, 1982). С точки зрения первой, интенция начать какую-либо деятельность (в том числе собственный бизнес) определяется следующими индивидуальными факторами: а) установками по отношению к деятельности (ожидаемые результаты); б) субъективными нормами (роль социального окружения при принятии решения); в) воспринимаемым контролем над деятельностью (perceived behavioral control оценка способности начать деятельность и достичь желаемого результата). Модель предпринимательских намерений предполагает три фактора, определяющих решение начать предпринимательскую деятельность: а) предпочтение предпринимательской деятельности (perceived desirability); б) предрасположенность к действиям (propensity to act); в) вера в осуществимость задуманного проекта (perceived feasibility). По этой теме осуществлен ряд исследований (Teixeira et al., 2018; Широкова и др., 2015; и др.).

Несмотря на распространенность данных подходов, исследователи отмечают необходимость расширения традиционных моделей для изучения предпринимательских намерений и процесса начала предпринимательской деятельности. Обращается внимание на роль целеполагания, мотивации и ценностей акторов в принятии решения о создании бизнеса (Fayolle et al., 2014; Tortia et al., 2020; Schlaegel, Koenig, 2013; Перикова и др., 2020). В частности, внимание к мотивам позволяет приблизиться к изучению связки «намерение-деятельность», поскольку конкретные причины создания проектов задают определенную траекторию их развития, направленность деятельности создателей.

\section{Шкала причин начала бизнес-проекта PSED}

Исходя из необходимости создания методики, учитывающей широкий спектр возможных мотивационных установок и содержательное наполнение инноваций, за основу была взята шкала «Причины начала бизнес-проектов», разработанная в рамках Панельного исследования предпринимательской динамики PSED. Ввиду многообразия возможных причин деятельности, шкала носит интегративный характер: она основана не на конкретной теоретической модели, а на метаанализе эмпирических исследований в сфере предпринимательства (Carter et al., 2004), в результате которого было выделено семь типов мотивов начала предпринимательской деятельности:

1) инновации - стремление создать что-то новое;

2) независимость - намерение быть более свободным и гибким при управлении своим временем;

3) экстернальная валидация - желание общественного признания и потребность в одобрении семьи, друзей и других членов сообщества;

4) роли - стремление продолжать семейные традиции или следовать примеру других;

5) финансовый успех - инструментальная ценность богатства, стремление к материальному благополучию и повышению финансового состояния;

6) самореализация - стремление к личностному росту и самовыражению; 
7) улучшение общества - стремление к социальным изменениям и помощи окружающим.

Каждому из мотивационных типов соответствует ряд суждений (таблица 1).

В связи с метааналитическим способом создания шкалы ее использование и интерпретация результатов в большой степени зависят от цели исследования и особенностей изучаемой группы. Выделенные типы мотивов нередко видоизменяются в зависимости от эмпирического объекта: мотив может складываться из сочетания нескольких типов, которые рассматриваются как отдельные в оригинальной версии шкалы (Quigley et al., 2015). Помимо этого, отсутствие заложенной теоретической модели дает исследователям свободу в плане выбора отдельных индикаторов в зависимости от специфики изучаемой группы (Xu et al., 2014).

\section{Методология}

\section{Процедура адаптащии шкаль PSED}

Процедура адаптации шкалы проводилась в несколько этапов (Muñiz et al., 2013; International Test Commission, 2017).

1. Перевод вопросника с применением техники двойного перевода.

2. Пилотаж переведенной анкеты. На теоретическом этапе были проведены пять интервью с экспертами, в ходе которых обсуждались релевантность и адекватность формулировок. В роли экспертов выступали исследователи в сфере образования. Затем проводился эмпирический этап пилотирования -

Распределение суждений по группам мотивов в шкале PSED

Таблица 1

\begin{tabular}{|l|l|}
\hline \multicolumn{1}{|c|}{ Название мотива } & \multicolumn{1}{c|}{ Индикаторы } \\
\hline Инновации & $\begin{array}{l}\text { Разработать идею нового продукта или услуги } \\
\text { Быть на передовом крае образовательных технологий } \\
\text { Развить свою личность }\end{array}$ \\
\hline Независимость & $\begin{array}{l}\text { Работать так, как я считаю нужным } \\
\text { Быть более гибким в своем графике работы }\end{array}$ \\
\hline $\begin{array}{l}\text { Экстернальная валида- } \\
\text { ция }\end{array}$ & $\begin{array}{l}\text { Заслужить уважение друзей } \\
\text { Повысить свой статус } \\
\text { Чего-то добитья, получить признание }\end{array}$ \\
\hline Роли & Продолжать семейное дело \\
\hline Финансовый успех & $\begin{array}{l}\text { Повысить собственный заработок } \\
\text { Построить бизнес, который смогли бы унаследовать мои дети } \\
\text { Иметь устойчивое финансовое положение, независимость } \\
\text { Стать состоятельным человеком }\end{array}$ \\
\hline Самореализация & $\begin{array}{l}\text { Испытать себя } \\
\text { Реализовать свое личное ви́дение, понимание }\end{array}$ \\
\hline Улучшение общества & $\begin{array}{l}\text { Запустить социальные перемены } \\
\text { Улучшить общество или его часть } \\
\text { Помочь другим людям }\end{array}$ \\
\hline
\end{tabular}


10 когнитивных интервью с инноваторами в образовании, в которых использовались техники пересказа вопроса, размышлений вслух и двойного интервью.

3. Пилотный онлайн-опрос. Он проводился для определения основных паттернов ответов и исключения вопросов, которые получили большое количество средних или однообразных ответов.

4. Основной этап сбора данных (апрель-май 2015). В ходе онлайн-опроса было опрошено 437 участников Конкурса инноваций в образовании (далее КИвО)․․ Тип выборки - невероятностная сплошная стихийная.

Инноваторы в образовании представляют собой широкую общность, включающую сотрудников как образовательных, так и сторонних организаций, что в значительной степени затрудняет поиск универсальных индикаторов их идентификации. В этой связи КИвО представляется удачной платформой для сбора данных, поскольку позволяет однозначно зафиксировать причастность каждого участника к инновационной деятельности в образовании на основании факта его участия в конкурсе. Аналогичный подход был использован в исследованиях инноваторов в областях технологий и спорта (Hellström et al., 2002). Исходя из этого, апробация шкалы на этой группе позволяет обеспечить ее валидность при изучении других представителей генеральной совокупности - акторов, реализующих собственные инициативы в образовательной сфере.

\section{Стратегия анализа мотивационной структурь}

На первом шаге анализа использовались процедуры разведывательного факторного анализа (РФА). С помощью РФА была выявлена мотивационная структура группы инноваторов в образовании, основанная на собранных данных, и оценено соответствие структуры, полученной на российской выборке, структуре, предложенной авторами опросника PSED.

На втором этапе использовался метод конфирматорного факторного анализа (КФА). С помощью КФА была уточнена первоначальная модель, полученная в результате РФА, и статистически подтверждена итоговая мотивационная структура.

\section{Результаты}

\section{Факторная структура русскоязычной версии опросника PSED}

\section{Поиск мотивационной структуры инноваторов с помощью РФА}

В ходе анализа ответов респондентов на вопросы о причинах создания инновационных проектов в ряде суждений было обнаружено значительное

\footnotetext{
${ }^{1}$ КИвО - крупнейший российский отраслевой конкурс инновационных образовательных проектов широкой направленности. Сбор данных проводился в период после окончания приема заявок на конкурс до объявления его результатов путем рассылки опроса на электронные адреса участников. Приглашались все участники без ограничений по количеству человек от одной команды.
} 
количество пропусков. Переменные «Продолжать семейное дело» и «Построить бизнес, который смогли бы унаследовать мои дети» (38 и 25\% пропусков соответственно) были исключены из финальной версии шкалы. Это связано с содержательными моментами: вероятно, суждения оказались нерелевантными деятельности инноваторов и по этой причине вызвали затруднения. РФА методом максимального правдоподобия проводился на выборке заполненных кейсов $(\mathrm{N}=286)$.

Тестировались модели с ортогональным и косоугольным вращением. В качестве итоговой была выбрана четырехфакторная модель с 16 суждениями и косоугольным вращением Promax (таблицы 2,3 ). При выборе вращения учитывались как статистические критерии, так и теоретические предположения о

Таблища 2

Статистики модели

\begin{tabular}{|l|c|}
\hline \multicolumn{1}{|c|}{ Статистика } & Значение \\
\hline КМО & 0.832 \\
\hline Тест сферичности Бартлетта & $\chi^{2}=1579, \mathrm{df}=120, p=0.000$ \\
\hline Процент общей объясненной дисперсии & $59 \%$ \\
\hline
\end{tabular}

Таблица 3

Результаты РФА

\begin{tabular}{|l|c|c|c|c|c|}
\hline \multicolumn{1}{|c|}{ Виды мотивации } & $\mathbf{1}$ & $\mathbf{2}$ & $\mathbf{3}$ & $\mathbf{4}$ & Общность \\
\hline Помочь другим людям & 0.75 & & & & 0.54 \\
\hline Запустить социальные перемены & 0.79 & & & & 0.66 \\
\hline Улучшить общество или его часть & 0.82 & & & & 0.69 \\
\hline Реализовать свое личное ви́дение, понимание & & 0.76 & & & 0.60 \\
\hline Работать так, как я считаю нужным & & 0.68 & & & 0.45 \\
\hline $\begin{array}{l}\text { Быть на передовом крае образовательных } \\
\text { технологий }\end{array}$ & & 0.66 & & & 0.46 \\
\hline Разработать идею нового продукта или услуги & & 0.57 & & & 0.41 \\
\hline Быть более гибким в своем графике работы & & & & 0.48 & 0.47 \\
\hline Повысить собственный заработок & & & & 0.87 & 0.78 \\
\hline $\begin{array}{l}\text { Иметь устойчивое финансовое положение, } \\
\text { независимость }\end{array}$ & & & & 0.89 & 0.78 \\
\hline Стать состоятельным человеком & & & & 0.86 & 0.79 \\
\hline Заслужить уважение друзей & & & 0.75 & & 0.57 \\
\hline Испытать себя & & & 0.71 & & 0.47 \\
\hline Чего-то добиться, получить признание & & & 0.70 & & 0.60 \\
\hline Развить свою личность & & & 0.65 & & 0.50 \\
\hline Повысить свой статус & & & 0.64 & & 0.60 \\
\hline \hline 1. Значимость для общества & - & 0.31 & 0.20 & 0.07 & \\
\hline 2. Инновации и креативность & & - & 0.42 & 0.35 & \\
\hline 3. Самореализация и достижение & & - & 0.48 & \\
\hline 4. Финансы и независимость & & - & \\
\hline
\end{tabular}


полимотивированности деятельности и, следовательно, коррелированности получаемых факторов.

\section{Подтверждение мотивационной структуры инноваторов с помощью КФА}

Выбранное четырехфакторное решение было протестировано с помощью КФА методом максимального правдоподобия. Первоначальная модель была изменена в соответствии с содержательными и статистическими соображениями (значениями модификационных индексов). Необходимость каждой модификации проверялась тестом на сравнение хи-квадратов двух вложенных моделей.

Добавлены корреляции между всеми мотивами, что значительно улучшило значения всех статистик согласия (модель 1 в таблице 4 и рисунок 1). Это подтверждает корректность выбора косоугольного решения в РФА: создание

Статистики согласия моделей КФА

Таблица 4

\begin{tabular}{|l|c|c|c|}
\hline Статистики согласия & Модель 1 & Модель 2 & Модель 3 \\
\hline$\chi^{2}$ & 210 & 193 & 178 \\
\hline Значимость $\chi^{2}$ & 0.000 & 0.000 & 0.000 \\
\hline $\mathrm{df}$ & 98 & 97 & 96 \\
\hline SRMR & 0.06 & 0.06 & 0.06 \\
\hline CFI & 0.93 & 0.94 & 0.95 \\
\hline TLI & 0.91 & 0.92 & 0.93 \\
\hline RMSEA & 0.06 & 0.06 & 0.06 \\
\hline
\end{tabular}

Итоговая модель КФА

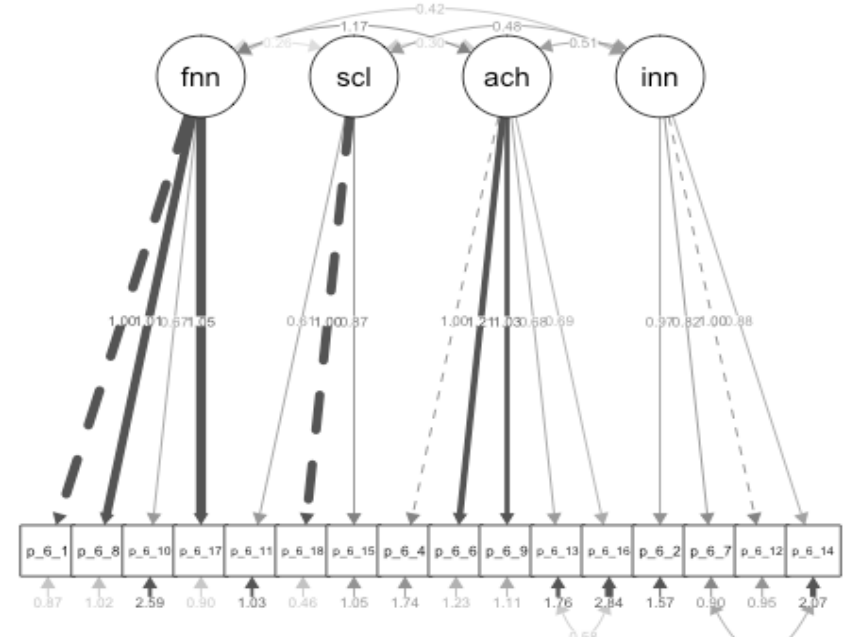


образовательных проектов - полимотивированная деятельность, не заданная одним направлением.

Добавлены корреляции остатков переменных внутри факторов: «Развить свою личность» и «Испытать себя» в факторе «Самореализация и достижение» (модель 2), «Разработать идею нового продукта или услуги» и «Работать так, как я считаю нужным» в факторе «Инновации и креативность» (модель 3). Модель 3 в высокой степени согласуется с данными и была выбрана в качестве итоговой (рисунок 1).

\section{Интерпретация полученных факторов}

Структура шкалы включает четыре фактора, каждый из которых соответствует мотиву создания образовательных проектов: «Значимость для общества», «Инновачии и креативность», «Самореализация и достижение» $и Ф и-$ нансы и независимость». Мотивы отражают определенные стремления и цели инноваторов, задавая содержание и направленность их образовательных проектов. Некоторые факторы обнаруживают высокую корреляцию между собой (таблица 3), однако содержательно каждая группа вопросов интерпретируется как отдельный мотив начала инновационных проектов в образовании.

Выраженность каждого фактора - это усредненное значение входящих в него суждений, которые оценивались респондентами по семибалльной шкале². Середина шкалы, отражающая среднюю выраженность мотива, равняется 3.5.

\section{Мотив «Значимость для общества» (3 суждения)}

Фактор отражает причины создания инновационных проектов, связанные с улучшением жизни окружающих и образовательной системы в целом.

Высокий балл по данному фактору свидетельствует о том, что для инноваторов важно привносить позитивные социальные изменения с помощью своего проекта. Низкий балл отражает низкую значимость просоциальных целей при создании образовательного проекта.

\section{Мотив «Инновачии и креативность» (4 суждения)}

Фактор отражает причины начала проектов, связанные со стремлением и готовностью быть на передовом крае инноваций, предлагать системе образования оригинальные идеи для ее модернизации и задействовать свою креативность в работе над проектом.

Высокий балл по этому фактору свидетельствует о том, что создание проекта рассматривается как способ проявления креативности и инновационности. Низкий балл - о том, что при создании проекта инноватор не руководствуется установкой на принципиальную новизну и творческий характер своей идеи.

\footnotetext{
${ }^{2}$ Мы используем средние значения входящих в фактор суждений вместо предсказанных факторных значений (factor scores) для большей интерпретируемости факторов (Grice, 2001). Расчет корреляций Пирсона показал тесную связь между предсказанными факторными значениями и средними значениями (>0.95).
} 


\section{Мотив «Самореализащия и достижение» (5 суждений)}

Фактор отражает причины создания образовательных проектов, связанные с актуализацией собственного потенциала, повышением статуса, в том числе через общественное признание важности проекта.

Высокий балл свидетельствует о том, что создание проекта связано в том числе с важностью личностного роста и самореализацией для автора проекта. Низкий балл отражает невысокую роль этих целей.

\section{Мотив «Финансы и независимость» (4 суждения)}

Фактор отражает причины создания проектов, связанные с достижением большей личной независимости и материального благополучия.

Высокий балл по данному фактору свидетельствует о том, что при создании проекта инноватор руководствуется стремлением сделать свою жизнь более гибкой и свободной, повысить собственную эффективность и добиться финансовой стабильности. Низкий балл свидетельствует о низкой значимости целей, связанных с повышением материального достатка и достижением независимости.

\section{Факторная структура шкаль PSED в оригинальной (англоязычной) и русскоязычной версиях}

По результатам анализа, мотивационная структура инноваторов в образовании отличается от заложенной в PSED и обнаруженной у группы социальных предпринимателей (Carter et al., 2004; Quigley et al., 2015). Мотив «Значимость для общества» соответствует мотиву улучшения общества в исследовании PSED. Мотив «Инновации и креативность» представляет собой сочетание мотивов инноваций, независимости и самореализации. Мотив «Самореализация и достижение» вобрал элементы мотивов экстернальной валидации, самореализации и инноваций по PSED. Причем два фактора в русскоязычной методике включили в себя сужения из факторов инноваций и самореализации оригинальной методики, они разделились содержательно. В первый фактор «Инновации и креативность» вошли суждения, в большей степени отражающие стремление инноватора проявить свою креативность и быть на передовом крае науки и инноваций. В то время как ко второму «Самореализация и достижение» - в большей степени относятся суждения, отражающие именно стремление к реализации потенциала. Другие суждения в этом факторе относятся к фактору экстернальной валидации, что позволяет дать этому фактору предложенную нами содержательную интерпретацию. Мотив «Финансы и независимость» включает элементы мотивов независимости и финансового успеха. Таким образом, инноваторы в образовании как члены принципиально иной социальной группы характеризуются специфической мотивационной структурой, которая отражает широту направленности их деятельности, несводимой только к предпринимательской. Создание проектов может быть связано как с личностными устремлениями, так и с желанием позитивных изменений на локальном и системном уровнях. 


\section{Проверка надежности опросника}

В дополнение к результатам КФА, надежность полученной структуры была проверена с помощью коэффициента согласованности альфа Кронбаха (таблица 5). Оценка надежности всех переменных одновременно не проводилась, так как подшкалы рассматриваются отдельно друг от друга. Исходя из результатов, факторы «Финансы и независимость», «Самореализация и достижение» и «Значимость для общества» обладают высокой надежностью: суждения внутри каждого из этих факторов хорошо согласованы между собой.

Фактор «Инновации и креативность» имеет более низкое значение. Детальный анализ этой подшкалы показал низкую корреляцию между суждениями «Работать так, как я считаю нужным» (№ 15) и «Разработать идею нового продукта или услуги» (№ 13). Попарные коэффициенты корреляций внутри фактора отражены в таблице 6. Удаление суждения № 15 привело к небольшому увеличению альфы (с 0.59 до 0.61). Тем не менее оно значимо коррелирует с остальными в подшкале. Также результаты факторного анализа методом главных компонент отдельно на четырех суждениях этого фактора свидетельствуют о наибольшей оптимальности однофакторной структуры исходя из критерия Кайзера и из высоких факторных нагрузок всех суждений. Исключение переменной № 15 привело к менее интерпретируемой модели. Учитывая статистические результаты КФА и дополнительных проверок, в целом удовлетворительную величину коэффициента согласованности и содержательную важность суждения, было решено сохранить первоначальное наполнение данного фактора.

Таблица 5

Показатели надежности факторов русскоязычной версии опросника PSED

\begin{tabular}{|c|l|c|}
\hline № шкалы & \multicolumn{1}{|c|}{ Название фактора } & Альфа \\
\hline 1 & Финансы и независимость & 0.84 \\
\hline 2 & Самореализация и достижение & 0.78 \\
\hline 3 & Значимость для общества & 0.72 \\
\hline 4 & Инновации и креативность & 0.59 \\
\hline
\end{tabular}

Таблица 6

Коэффициенты корреляции суждений внутри фактора «Инновации и креативность»

\begin{tabular}{|c|c|c|c|}
\hline № суждения & $\mathbf{1 4}$ & $\mathbf{1 3}$ & $\mathbf{1 6}$ \\
\hline 14 & 1 & $0.313^{* *}$ & $0.340^{* *}$ \\
\hline 13 & $0.313^{* *}$ & 1 & $0.375^{* *}$ \\
\hline 16 & $0.340^{* *}$ & $0.375^{* *}$ & 1 \\
\hline 15 & $0.237^{* *}$ & 0.068 & $0.339^{* *}$ \\
\hline
\end{tabular}

$* * p<0.01$. 


\section{Заключение}

Несмотря на то что мотивация начала низовой инновационной деятельности в образовании, т.е. включения новой группы людей в существующую сферу общественной жизни, является одним из ключевых элементов портрета таких акторов, ощущается дефицит инструментов для измерения их мотивации. Для заполнения этой ниши в ходе исследования была разработана методика изучения мотивации к созданию инновационных проектов в образовании. Она представляет собой шкалу из 16 суждений, каждое из которых соответствует определенным причинам создания собственного проекта. Шкала позволяет оценить выраженность четырех мотивационных установок начала инновационной деятельности: «Значимость для общества», «Инновации и креативность», «Самореализация и достижение» и «Финансы и независимость».

Факторный анализ шкалы, проведенный в рамках классического подхода совмещения методов РФА и КФА, показал, что русскоязычной версии соответствует четырехфакторная структура. Дополнительный анализ оценки внутренней согласованности подшкал (альфа Кронбаха, корреляционный и факторный анализ) показал в целом высокую надежность обнаруженной структуры.

Разработанная методика может использоваться в исследовательских целях для изучения мотивов создания проектов или мотивации участия в деятельности по развитию социально значимых сфер в группах, схожих с инноваторами в образовании: низовых и институциональных инноваторов, социальных предпринимателей, резидентов стартап-акселераторов и т.п.

Кроме решения исследовательских задач, шкала обладает потенциалом прикладного использования. С практической точки зрения методика может применяться лицами, занимающимися разработкой политики в сфере поддержки индивидуальных проектов и создания среды, благоприятной для развития низовых инноваций в образовании и других социальных сферах. Понимание мотивов позволит предлагать диверсифицированные способы поддержки проектов и создания среды в зависимости от выявленной мотивации.

\section{Литература}

Богатырева, К., Широкова, Г. (2017). От предпринимательских намерений - к созданию бизнеса: опыт российских студентов. Форсайт, 11(3), 25-36.

Королева, Д. О., Хавенсон, Т. Е., Андреева, А. А. (2017). Ландиафт образовательных инноваций: содержание и структура. М.: НИУ ВШЭ.

Кузьминов, Я. И., Фрумин, И. Д. (ред.). (2018). Двенадиать решений для нового образования (Доклад Центра стратегических разработок и Высшей школы экономики). М.: НИУ ВШЭ. Режим доступа: https://publications.hse.ru/books/218061780

Перикова, Е. И., Атаманова, И. В., Богомаз, С. А. (2020). Специфика психологической готовности к инновационной деятельности молодежи Санкт-Петербурга и Томска. Science for Education Today, 10(1), 62-78. doi:10.15293/2658-6762.2001.04

Хавенсон, Т. Е., Королева, Д. О., Лукина, А. А. (2018). Акторы образовательных инноваций: Ценности и мотивация. М.: НИУ ВШЭ. Режим доступа: https://publications.hse.ru/books/227119446 
Широкова, Г. В., Цуканова, Т. В., Богатырева, К. А. (2015). Университетская среда и предпринимательская активность студентов: Роль бизнес-опыта и предпринимательской самоэффективности. Вопросы образования, 3, 171-207.

Ссылки на зарубежные источники см. в разделе References после англоязычного блока.

Приложение

Методика измерения мотивов инноваторов в образовании

Ниже приведен список суждений, составляющих опросник, и инструкция, предваряющая вопросы. Суждения должны предъявляться респондентам в случайном порядке.

\section{Инструкция}

Ниже приведена серия утверждений, отражающих различные причины реализации инновационных проектов. В каждом из них есть 7 возможных ответов. Ответы варьируются от 1 - причина совсем не важна до 7 - причина очень важна, 4 - отражает нейтральное отношение. Отметьте, пожалуйста, насколько каждая причина важна лично для вас. Отметьте, пожалуйста, только один вариант, который наиболее точно отражает ваше мнение по отношению к утверждению. Пожалуйста, ответьте на все вопросы.

\begin{tabular}{|c|c|c|c|c|c|c|c|c|}
\hline \multirow[t]{2}{*}{ № } & \multirow[t]{2}{*}{ Утверждение } & \multicolumn{7}{|c|}{$\begin{array}{c}\text { Причина совсем не важна (1) - } \\
\text { причина очень важна (7) }\end{array}$} \\
\hline & & & & & & & & \\
\hline 1. & Чего-то добиться, получить признание & 1 & 2 & 3 & 4 & 5 & 6 & 7 \\
\hline 2. & Развить свою личность & 1 & 2 & 3 & 4 & 5 & 6 & 7 \\
\hline 3. & Заслужить уважение друзей & 1 & 2 & 3 & 4 & 5 & 6 & 7 \\
\hline 4. & Повысить свой статус & 1 & 2 & 3 & 4 & 5 & 6 & 7 \\
\hline \multirow[t]{2}{*}{5.} & Испытать себя & 1 & 2 & 3 & 4 & 5 & 6 & 7 \\
\hline & \multicolumn{8}{|l|}{ Мотив «Финансы и независимость» } \\
\hline 6. & Повысить собственный заработок & 1 & 2 & 3 & 4 & 5 & 6 & 7 \\
\hline 7. & $\begin{array}{l}\text { Иметь устойчивое финансовое положение, } \\
\text { независимость }\end{array}$ & 1 & 2 & 3 & 4 & 5 & 6 & 7 \\
\hline 8. & Быть более гибким в своем графике работы & 1 & 2 & 3 & 4 & 5 & 6 & 7 \\
\hline \multirow[t]{2}{*}{9.} & Стать состоятельным человеком & 1 & 2 & 3 & 4 & 5 & 6 & 7 \\
\hline & \multicolumn{8}{|l|}{ Мотив «Значимость для общества» } \\
\hline 10. & Запустить социальные перемены & 1 & 2 & 3 & 4 & 5 & 6 & 7 \\
\hline 11. & Улучшить общество или его часть & 1 & 2 & 3 & 4 & 5 & 6 & 7 \\
\hline \multirow[t]{2}{*}{12.} & Помочь другим людям & 1 & 2 & 3 & 4 & 5 & 6 & 7 \\
\hline & \multicolumn{8}{|l|}{ Мотив «Инновации и креативность» } \\
\hline 13. & Разработать идею нового продукта или услуги & 1 & 2 & 3 & 4 & 5 & 6 & 7 \\
\hline 14. & Быть на передовом крае образовательных технологий & 1 & 2 & 3 & 4 & 5 & 6 & 7 \\
\hline 15. & Работать так, как я считаю нужным & 1 & 2 & 3 & 4 & 5 & 6 & 7 \\
\hline 16. & Реализовать свое личное ви́дение, понимание & 1 & 2 & 3 & 4 & 5 & 6 & 7 \\
\hline
\end{tabular}




\title{
Подсчет и интерпретация результатов
}

Все утверждения опросника имеют прямую кодировку, поэтому вариант ответа, выбранный респондентом, является его баллом, полученным за соответствующий вопрос (от 1 до 7). Подсчет итоговых баллов происходит отдельно для каждого фактора, поскольку мотивы являются многомерным конструктом: разные мотивы могут иметь разную значимость у одного респондента. Соответственно общий балл по всему опроснику не рассчитывается.

Хавенсон Татьяна Евгеньевна - старший научный сотрудник, лаборатория инноваций в образовании, Институт образования, Национальный исследовательский университет «Высшая школа экономики», $\mathrm{PhD}$ наук об образовании.

Сфера научных интересов: инновации в образовании, измерения в социальных науках,доказательная образовательная политика.

Контакты: tkhavenson@hse.ru

Лукина Анастасия Андреевна - младший научный сотрудник, Центр социологии культуры, Институт образования, Национальный исследовательский университет «Высшая школа экономики».

Сфера научных интересов: измерения в социальных науках, формальные методы изучения культуры, культурсоциология.

Контакты: aalukina@hse.ru

\section{Measuring Motivation for Innovation in Education: Concept Definition and Scale Validation}

\author{
T.E. Khavenson ${ }^{\text {a }}$ A.A. Lukina ${ }^{a}$
}

${ }^{a}$ HSE University, 20 Myasnitskaya Str., Moscow, 101000, Russian Federation

\begin{abstract}
The reasons for innovations inside and outside the education system have attracted much research interest over the past decades. However, there is still a lack of methodological tools to measure motives of actors coming from inside and outside the system to launch their innovative projects. The article describes approaches to measure the construct "motivation for innovative activity," as well as the results of adaptation of the scale aimed at measuring motives for creation of innovative educational projects. The tool is based on the scale, constructed in English, "Reasons to create business projects" (PSED). In this research the scale was translated into Russian, adapted for the group of innovators in education and validated within a classical framework of combining exploratory and confirmatory factor analysis (EFA and CFA, respectively). Additionally, the subscales were evaluated in terms of internal coherence. The Russian and the English version of the scale were compared, and each subscale was interpreted. Adaptation was performed on the sample of innovators in education, that is, the participants of Competition of Innovations in Education $(\mathrm{N}=286)$. The final scale includes 16 statements and allows to evaluate the intensity of four motivational attitudes towards innovative activity: "Social significance," "Innovations and creativity," "Self-realization and achievement," "Finance and autonomy." The
\end{abstract}


identified motives reflect certain endeavors and goals of innovators and determine the content and orientation of their educational projects. The instrument can be used for both research and practice purposes to explore motivation of proactive actors in education, as well as similar groups involved in the development of public social spheres. The full version of the scale is presented in the appendix and contains the instructions for respondents and scoring criteria for four subscales.

Keywords: motivation towards innovations, PSED, scale adaptation, measuring motives of innovative activity.

\section{References}

Ajzen, I. (1991). The theory of planned behavior. Organizational Behavior and Human Decision Processes, 50(2), 179-211.

Ajzen, I. (2002). Perceived behavioral control, self-efficacy, locus of control, and the theory of planned behavior. Journal of Applied Social Psychology, 32(4), 665-683. doi:10.1111/j.15591816.2002.tb00236.x

Attali, M. O., \& Yemini, M. (2017). Initiating consensus: stakeholders define entrepreneurship in education. Educational Reviere, 69(2), 140-157. doi:10.1080/00131911.2016.115345

Battilana, J., Leca, B., \& Boxenbaum, E. (2009). How actors change institutions: Towards a theory of institutional entrepreneurship. The Academy of Management Annals, 3(1), 65-107. doi:10.1080/19416520903053598

Bogatyreva, K., \& Shirokova, G. (2017). From entrepreneurial aspirations to founding a business: The case of Russian students. Foresight and STI Governance, 11(3), 25-36. doi:10.17323/25002597.2017.3.25.36. Retrieved from https://foresight-journal.hse.ru/data/2017/09/27/ 1172894856/Shirokova\%2025-36.pdf

Caringal-Go, J. F., \& Hechanova, M. R. M. (2018). Motivational needs and intent to stay of social enterprise workers. Journal of Social Entrepreneurship, 9(3), 200-214. doi:10.1080/19420676.2018.1468352

Carsrud, A., \& Brännback, M. (2011). Entrepreneurial motivations: What do we still need to know? Journal of Small Business Management, 49(1), 9-26. doi:10.1111/j.1540-627x.2010.00312.x

Carter, N. M., Gartner, W. B., \& Shaver, K. G. (2004). Career reasons. In W. B. Gartner, K. G. Shaver, N. M. Carter, \& P. D. Reynolds (Eds.), Handbook of entrepreneurial dynamics: The process of business creation (pp. 142-152). Thousand Oaks, CA: Sage.

Deci, E. L., \& Ryan, R. M. (1985). Intrinsic motivation and self-determination in human behavior. New York, NY: Plenum.

Douglas, E., \& Prentice, C. (2019). Innovation and profit motivations for social entrepreneurship: A fuzzy-set analysis. Journal of Business Research, 99, 69-79. doi:10.1016/j.jbusres.2019.02.031

Elfving, J. (2008). Contextualizing entrepreneurial intentions: a multiple case study on entrepreneurial cognitions and perceptions. Turku, FI: Åbo Akademi förlag.

Fayolle, A., Liñán, F., \& Moriano, J. A. (2014). Beyond entrepreneurial intentions: values and motivations in entrepreneurship. International Entrepreneurship and Management Journal, 10(4), 679689. doi:10.1007/s11365-014-0306-7 
Frese, M., \& Gielnik, M. M. (2014). The psychology of entrepreneurship. Annual Review of Organizational Psychology and Organizational Behavior, 1(1), 413-438.

Ganguli, I., Huysentruyt, M., \& Le Coq, C. (2018). How do nascent social entrepreneurs respond to rewards? a field experiment on motivations in a grant competition. UMass Amherst Economics Working Papers, 258, 45.

Grice, J. W. (2001). Computing and evaluating factor scores. Psychological Methods, 6(4), 430-450. doi:10.1037/1082-989X.6.4.430

Hellström, T., Hellström, C., \& Berglund, H. (2002). The innovating self: Exploring self among a group of technological innovators. Journal of Managerial Psychology, 17(4), 267-286. doi:10.1108/02683940210428083

International Test Commission. (2017). The ITC guidelines for translating and adapting tests. Version, $1,20-12$.

Jones, J., Seet, P.-S., Acker, T., \& Whittle, M. (2021). Barriers to grassroots innovation: The phenomenon of social-commercial-cultural trilemmas in remote indigenous art centres. Technological Forecasting and Social Change, 164, 119583. doi:10.1016/j.techfore.2019.02.003

Khavenson, T. E., Koroleva, D. O., \& Lukina, A. A. (2018). Aktory obrazovatel'nykh innovatsii: Tsennosti i motivatsiya [Actors of educational innovations: Values and motivation]. Moscow: HSE Publishing House. Retrieved from https://publications.hse.ru/books/227119446 (in Russian)

Koroleva, D. O., Khavenson, T. E., \& Andreeva, A. A. (2017). Landshaft obrazovatel'nykh innovatsii: soderzhanie $i$ struktura [The landscape of educational innovations: the content and structure]. Moscow: HSE Publishing House. (in Russian)

Kuz'minov, Ya. I., \& Frumin, I. D. (Eds.). (2018). Dvenadtsat' reshenii dlya novogo obrazovaniya (Doklad Tsentra strategicheskikh razrabotok i Vysshei shkoly ekonomiki) [Twelve decisions for a new education (A report by the Centre of Strategic Developments and Higher School of Economics]. Mosciow: HSE Publishing House. Retrieved from https://publications.hse.ru/books/218061780 (in Russian)

Muńiz, J., Elosua, P., \& Hambleton, R. K. (2013). International Test Commission Guidelines for test translation and adaptation. Psicothema, 25(2), 151-157.

OECD. (2014). Measuring innovation in education: A new perspective, educational research and innovation. Paris: OECD.

Perikova, E. I., Atamanova, I. V., \& Bogomaz, S. A. (2020). Specific features of psychological readiness for innovative activity (with the main focus on young adults in St. Petersburg and Tomsk). Science for Education Today, 10(1), 62-78. doi:10.15293/2658-6762.2001.04 (in Russian)

Phelps, E. (2018). The dynamism of nations: toward a theory of indigenous innovation. Journal of Applied Corporate Finance, 30(3), 8-26.

Quigley, N. R., Newbert, S. L., \& Clark, K. D. (2015). The motivations underlying small business formation: An overview and empirical examination of self-efficacy and commitment. In S. L. Newbert (Ed.), Small business in a global economy: Creating and managing successful organizations (pp. 21-49). Denver, CO: Praeger.

Schlaegel, C., \& Koenig, M. (2013). Determinants of entrepreneurial intent: A meta-analytic test and integration of competing models. Entrepreneurship Theory and Practice, 38(2), 291-332. doi:10.1111/etap.12087

Seelos, C., \& Mair, J. (2005). Social entrepreneurship: Creating new business models to serve the poor. Business Horizons, 48(3), 241-246. 
Seyfang, D. G., \& Smith, D. A. (2007). Grassroots innovations for sustainable development: Towards a new research and policy agenda. Environmental Politics, 16(4), 584-603. doi:10.1080/09644010701419121

Shapero, A., \& Sokol, L. (1982). The social dimensions of entrepreneurship. In C.A. Kent, D. L. Sexton, \& K. H. Vesper (Eds.), Encyclopedia of entrepreneurship (pp. 72-90). Englewood Cliffs, NJ: Prentice-Hall.

Shirley, D. (2017). Accelerating educational change. Journal of Educational Change, 18(3), 257-262. doi:10.1007/s10833-017-9303-2

Shirokova, G. V., Tsukanova, T. V., \& Bogatyreva, K. A. (2015). University environment and student entrepreneurship: the role of business experience and entrepreneurial self-efficacy. Voprosy Obrazovaniya [Educational Studies Moscow], 3, 171-207. (in Russian)

Teixeira, S. J., Casteleiro, C. M. L., Rodrigues, R. G., \& Guerra, M. D. (2018). Entrepreneurial intentions and entrepreneurship in European countries. International Journal of Innovation Science, 10(1), 22-42. doi:10.1108/IJIS-07-2017-0062

Tortia, E. C., Degavre, F., \& Poledrini, S. (2020). Why are social enterprises good candidates for social innovation? Looking for personal and institutional drivers of innovation. Annals of Public and Cooperative Economics, 91(3), 459-477. doi:10.1111/apce.12265

Xu, Z., Robinson, J. A., Germak, A. J., \& Huang C. (2014, November). For Love or Money? Achievement Orientation, Non-monetary Focus, and the Nascent Social Entrepreneur. Paper presented at the ARNOVA's 43rd Annual Conference, Denver, CO.

Zahra, S. A., Gedajlovic, E., Neubaum, D. O., \& Shulman, J. M. (2009). A typology of social entrepreneurs: Motives, search processes and ethical challenges. Journal of Business Venturing, 24(5), 519-532.

Tatiana E. Khavenson - Associate Professor, Laboratory for Innovations, Institute of Education, HSE University, PhD in education.

Research Area: innovative behavior in education, measurement in social sciences, evidence-based education policy.

E-mail: tkhavenson@hse.ru

Anastasia A. Lukina - Junior Research Fellow, Centre for Cultural Sociology, Institute of Education, HSE University.

Research Area: measurement in social sciences, formal methods of cultural analysis, cultural sociology.

E-mail: aalukina@hse.ru 